\title{
Sensor Networks and Optimal Regular Covering of the Plane with Equal Sectors
}

\author{
A. Erzin \\ Sobolev Institute of Mathematics \\ Novosibirsk, Russia
}

\author{
N. Shabelnikova \\ Novosibirsk State University \\ Novosibirsk, Russia
}

\begin{abstract}
We suppose that in the sensor network, the sensing areas of the sensors are equal sectors, and consider the problem of regular covering of the plane with minimal number of identical sensors per unit area. In the regular cover, the plane is split into the equal regular polygons - "tiles" (equilateral triangles, squares or regular hexagons), and all the tiles are covered equally. We solved the problem for the special case when every sensor covers one tile, and all the sensors covering one tile are placed in one point.
\end{abstract}

\section{Keywords-sensor networks; regular coverage; optimization}

\section{INTRODUCTION}

In the wireless sensor networks (WSN) each sensor has a limited, as usually, non-renewable energy which is consumed particularly on the sensing and communication. Then the main goal in WSN is lifetime maximization [5, 8, 9, 10, 15, 17, 20, 23]. If only sensing energy consumption is taken into account, then the problem can be reduced to the construction of the least density covers [3, 5, 7, 16, 21, 23].

A cover of a plane region is fully specified if one knows the figures included in the cover, the location of each figure and its orientation. For example, if the figures in a cover are ellipses, then it is sufficient to know the semi-axes, the location of the centre and the slope of the axis of each ellipse [7].

Because of the variety of the covers, in the literature the regular covers are often considered. In [23] a classification of regular covers proposed. According to this classification the set $\operatorname{COVl}(p, q)$ consists of the covers, in each of which a plane region is split into the regular l-angle polygons $(l=3,4,6)$, and all the polygons are covered equally with $\mathrm{p}$ figures of $\mathrm{q}$ different types $(\mathrm{q} \leq \mathrm{p})$.

Interest to the regular covers is caused not only by their relative simplicity and convenience of analysis, but also by other considerations. In the regular cover the sensors are placed in the certain points. In fact, it is sometimes difficult to place the sensors in the specific locations. But even in the case when sensors are distributed randomly, the regular covers can help to estimate the bounds of WSN's lifetime [2, 22].

Frequently the disks are used as an area covered by a sensor [3-5, 8, 9, 11, 16, 19]. In [12] shown that the plane cover with equal disks, in which the centres of three overlapping disks are the vertices of the equilateral triangle, has a minimum density among all plane covers with equal disks. G. Fejes Töth proposed a cover of the plane with disks of two radii, the density of which tends to 1.0189 as the radius of small disks tends to zero [19]. The disadvantage of the proposed cover is unlimited number of small disks. In real systems, the number of disks per unit area must be limited and, moreover, the sizes of the disks in a cover should be comparable.

However, in practice, a sensor often covers an area different from the disk. Thus in a number of recent publications the ellipse and sector coverage models are considered $[1,6,7,9,13,14,18]$. In this paper we propose and analyze the regular covers with equal sectors in which the number of sectors per unit area is minimal. The problem of minimizing the number of sectors per unit area is closely related to the problem of the least dense coverage, but does not coincide with it completely. We found the optimal number of sectors covering one tile in the case when every sector is involved in covering only one tile, and the vertices of the sectors (the sensors) which cover one tile are located in one point.

Using sectors in the cover is still poorly studied subject matter, and there are a few publications on this topic $[1,6,9$, $10,13,14,18]$. The density of a cover with equal sectors does not dependent on the size of the sectors involved in the coverage, but depends on the ratio of the sector's area to the area of a tile. Given the cost of sensors, then naturally arises the problem of finding the plane cover with equal sectors in which the minimum number of sectors is used to cover a unit area. Moreover, due to the technical reasons, angle and radius of the sector often cannot take arbitrary values. Additionally, if we take into account the cost of installation and communication of the sensors, it should be preferred to place several sensors (the vertices of sectors) in the same site (point).

The foregoing consideration gives rise to the problem of constructing the regular plane covers with equal sectors with minimal number of sectors per unit area, where each sector involved in covering only one tile, and the vertices of sectors covering one tile are located at one point.

\section{MATHEMATICAL STATEMENT OF THE PROBLEM}

We consider a class of regular covers $\operatorname{COVl}(p, 1), l=3,4$, 6. In each cover a plane is tiled by regular l-angle polygons (tiles), and the identical sectors with angle $\alpha$ and radius $\mathrm{R}$ (hereinafter we denoted the sector by a pair $(\alpha, \mathrm{R})$ ) are used to cover the tiles. The parameters of sector take the values within the given limits $\alpha \in[\alpha 1, \alpha 2], \mathrm{R} \in[\mathrm{R} 1, \mathrm{R} 2], 0<\alpha 1 \leq \alpha 2 \leq \pi$; $0<\mathrm{R} 1 \leq \mathrm{R} 2<+\infty$, and every sector is used to cover only one tile. But one tile can be covered by several sectors. Since we consider the regular covers, then all the tiles are covered 
equally. It is required to cover a tile object to minimize the number of sectors per unit of covered area on conditions that the vertices of the sectors covering one tile are located at the same point. So, the problem is to find $\min \frac{k(\alpha, R)}{S(k(\alpha, R) \cdot \alpha, 1}$

$k(\alpha, R) \in \mathrm{Z}^{+} S(k(\alpha, R) \cdot \alpha, R)$ for any $\alpha \in[\alpha 1, \alpha 2]$ and $\mathrm{R} \in[\mathrm{R} 1$, $\mathrm{R} 2$ ], where $\mathrm{Z}+$ is the set of the positive integers, $k(\alpha, \mathrm{R})$ is the number of sectors $(\alpha, R)$ with vertices at one point, and these sectors are involved in covering one tile, and $S(k \alpha, R)$ is the maximum area of a tile inscribed in the sector ( $k \alpha, \mathrm{R})$ (a sector which is formed by $\mathrm{k}$ adjacent sectors with the angles $\alpha$, whose vertices are in the same point).

In this paper we present the results of study with different tiles and formulate the final result as a theorem, setting (for simplicity) $\alpha \mathrm{R} 2=\mathrm{S}=$ const. Then the problem can be rewritten as where $\mathrm{k}(\alpha)$ is the number of sectors covering one tile (triangular, square or hexagonal), and $\mathrm{S}(\mathrm{k} \alpha)$ is the maximum area of the tile inscribed in the sector $(k \alpha, \sqrt{S / \alpha})$.

$$
\sigma(\alpha)=\min _{k(\alpha) \in Z^{+}} \frac{k(\alpha)}{S(k(\alpha) \cdot \alpha)} .
$$

III. THE OPTIMAL NUMBER OF SECTORS COVERING ONE TILE In order to solve the problem (1), we first found the optimal coverage models for all possible tiles (triangular tile Figure. 1, square tile - Figure. 2 and hexagonal tile - Figure. 3).

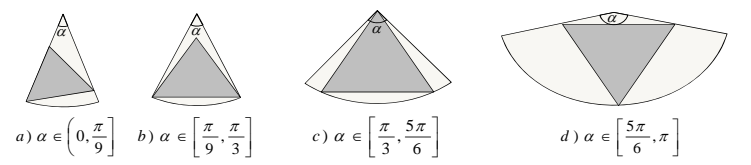

FIGURE 1. OPTIMAL COVERAGE MODELS FOR TRIANGLE TILE DEPENDING ON ANGLE.

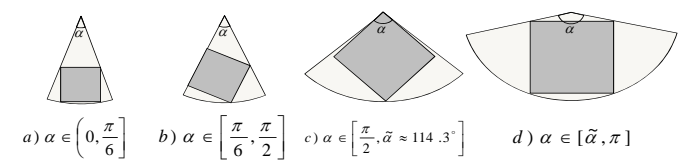

FIGURE 2. OPTIMAL COVERAGE MODELS FOR SQUARE TILE DEPENDING ON ANGLE.

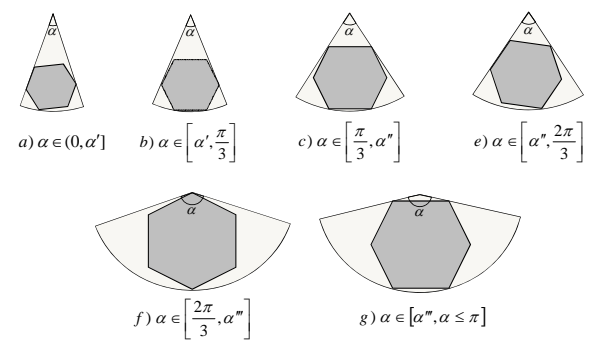

FIGURE 3. OPTIMAL COVERAGE MODELS FOR HEXAGONAL TILE DEPENDING ON ANGLE, WHERE $\alpha^{\prime} \approx 35.3^{\circ}, \alpha^{\prime \prime} \approx 92.6^{\circ}$, $\alpha^{\prime \prime \prime} \approx 135.5^{\circ}$
We proved the following principal theorem.

Theorem. Let $\alpha \in\left[\alpha_{1}, \alpha_{2}\right], 0<\alpha_{1} \leq \alpha_{2} \leq \pi$ and $\alpha R^{2}=S=$ const .

$$
\text { If } \alpha \in\left[\alpha_{1}, \alpha_{2}\right] \subseteq\left(0,35.2^{\circ}\right] \text {, then } \sigma(\alpha)=\min \left\{\frac{2 \alpha\lfloor\pi / 3 \alpha\rfloor}{S \sqrt{3}(1-\cos \lfloor/ 3 \alpha \alpha \alpha)}, \frac{4 \pi / 3 \alpha}{S \sqrt{3}}\right\} \text {; }
$$

else if $\alpha \in\left[\alpha_{1}, \alpha_{2}\right] \subseteq\left[35.2^{\circ}, \pi / 3\right]$, then

$\sigma(\alpha)=\min \left\{\frac{2 \alpha\lfloor\pi / 3 \alpha\rfloor}{S \sqrt{3}(1-\cos \pi / 3 \alpha \alpha \alpha)}, \frac{4 \pi / 3 \alpha}{S \sqrt{3}}, \frac{2 \alpha}{S \sqrt{3}(1-\cos \alpha)}, \frac{\alpha}{3 \sqrt{3} S}\left(1+\left(2 \sqrt{3}+\frac{1}{\tan \alpha}\right)^{2}\right)\right\} ;$

else if $\alpha \in\left[\alpha_{1}, \alpha_{2}\right] \subseteq\left[\pi / 3,73.8^{\circ}\right]$, then $\sigma(\alpha)=\frac{4 \alpha}{S \sqrt{3}}$;

else if $\alpha \in\left[\alpha_{1}, \alpha_{2}\right] \subseteq\left[73.8^{\circ}, 89.3^{\circ}\right]$, then

$\sigma(\alpha)=\frac{\alpha}{6 \sqrt{3} S}\left(1+\left(2 \sqrt{3}+1 / \tan \frac{\alpha}{2}\right)^{2}\right)$

else if $\alpha \in\left[\alpha_{1}, \alpha_{2}\right] \subseteq\left[89.3^{\circ}, \pi / 2\right]$, then

$\sigma(\alpha)=\frac{\alpha}{S}\left(1+\left(1+\frac{1}{\tan \alpha}\right)^{2}\right)$

else if $\alpha \in\left[\alpha_{1}, \alpha_{2}\right] \subseteq\left[\pi / 2,90.9^{\circ}\right]$, then $\sigma(\alpha)=2 \alpha / S$;

else if $\alpha \in\left[\alpha_{1}, \alpha_{2}\right] \subseteq\left[90,9^{\circ}, 179,6^{\circ}\right]$, then $\sigma(\alpha)=1 / S_{h}(\alpha)$;

else if $\alpha \in\left[\alpha_{1}, \alpha_{2}\right] \subseteq\left[179.6^{\circ}, \pi\right]$, then

$\sigma(\alpha)=\frac{\alpha}{4 S}\left(1+\left(2+1 / \tan \frac{\alpha}{2}\right)^{2}\right)$

where $S_{h}(\alpha)$ is the maximal square of regular hexagon inscribed in the sector $(\alpha, \sqrt{S / \alpha})$.

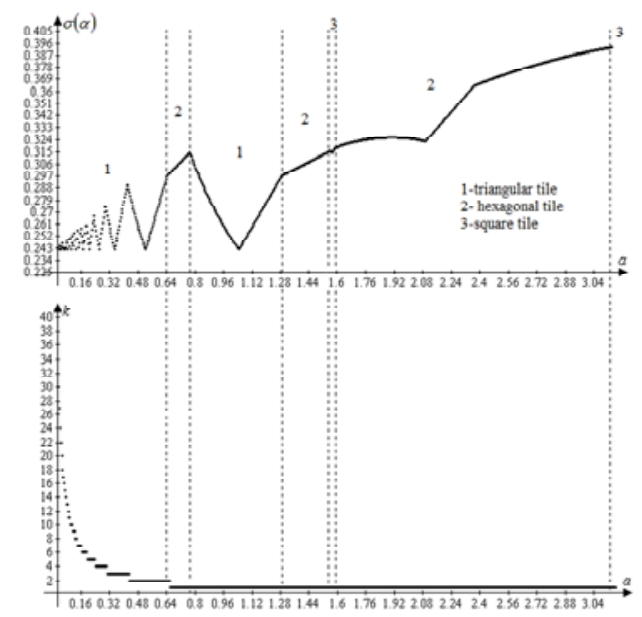

FIGURE 4. BEHAVIOR OF THE OBJECTIVE FUNCTION, AND THE OPTIMAL NUMBER OF SECTORS COVERING ONE TILE.

So, if we know the angle $\alpha$, then we can calculate the values of the expressions in the theorem and to compare them. Then the time complexity to find the optimal value of the 
objective function $\sigma(\alpha)$ (and to solve the problem (1)) is $\mathrm{O}(1)$ (i.e. a constant)!

In Figure. 4 we illustrate the results of the theorem. The upper part of Figure. 4 shows the behavior of the objective function and the optimal form of tiles, and the lower part of the figure indicates the optimal number of sectors covering one tile $\mathrm{k}(\alpha)$.

\section{CONCLUSION}

In this paper we investigated the regular plane coverage models with identical sectors, where the entire plane is divided into the regular 3-, 4- and 6-angle polygons (tiles), each sector involved in covering of exactly one tile, and the vertices of the sectors covering one tile are one common point.

The optimal models of tile covering by one sector that depend on the angle of the sector are found. These models allowed us to solve the problem of constructing the regular covers of a plane, wherein the number of sectors per unit area is minimal.

In this paper we considered the unbounded area (the whole plane) which is covered with equal sectors. In practice the area may be bounded, and the corresponding coverage problems are rather complicated. In the nearest future we are planning to consider the regular covers of the stripe and rectangle based on our experience of covering the stripe with circles and ellipses $[3,7]$.

\section{ACKNOWLEDGMENT}

The reported study was partially supported by Russian Fund of Basic Research, projects No. 13-07-00139. We are grateful to the Novosibirsk State University for providing us the Maple 17.02 package.

\section{REFEREENCES}

[1] Ai J., Abouzeid A.A. Coverage by directional sensors in randomly deployed wireless sensor networks // J. of Combinatorial Optimization, No. 11, 21-41, 2006.

[2] Aldyn-ool T., Erzin A., Zalyubovskiy V. The coverage of a planar region by randomly deployed sensors // Vestnik, Quart. J. of Novosibirsk State Univ., Series: Math., mech. and informatics (in Russian), 10(4), 7-25, 2010.

[3] Astrakov S., Erzin A. Efficient band monitoring with sensors outer positioning // Optimization: A Journal of Mathematical Programming and Operations Research, 62(10), 1367-1378, 2013.

[4] Boroczky K. J. Finite packing and covering. Cambridge University Press, Cambridge, 2004.

[5] Cardei M. Improving network lifetime using sensors with adjustable sensing ranges // Int. J. of Sensor Networks, 1(1/2), 41-49, 2006.

[6] Deshpande N., Shaligram A. Energy saving in wsn with directed connectivity // Wireless Sensor Network, No. 5, 121-126, 2013.

[7] Erzin A., Astrakov S. Covering a plane with ellipses // Optimization: A Journal of Mathematical Programming and Operations Research, 62(10), 1357-1366, 2013.

[8] Fan G., Jin S. Coverage problem in wireless sensor network: A survey. // Journal of Networks, 5(9), 1033-1040, 2010.

[9] Guvensan M.A., Yavuz A.G. On coverage issues in directional sensor networks. a survey // Ad Hoc Networks, No. 9, 1238-1255, 2011.

[10] Han X., Cao X., Lloyd E., Shen C.-C. Deploying directional sensor networks with guaranteed connectivity and coverage // 5th Annual IEEE Communications Society Conference on Sensor, Mesh and Ad Hoc Communications and Networks, 153-160, 2008.

[11] Ismailescu D., Kim B. Packing and covering with centrally symmetric convex disks // Discrete \& Computational Geometry, No. 51, 495-508, 2014.
[12] Kershner R. The number of circles covering a set // American J. of Mathematics, 62(3), 665-671, 1939.

[13] Li J., Wang C.R., Huang H.P., Sun L.J. Voronoi based area coverage optimization for directional sensor networks // Wireless Sensor Network, No. 1, 417-424, 2009.

[14] Liu L., Zhang X., Ma H. Exposure-path prevention in directional sensor networks using sector model based percolation // IEEE Int. Conf. on Communications, 274-278, 2009.

[15] Nguyen N., Zalyubovskiy V., Ha M., Le T., Choo H. Energy-efficient models for coverage problem in sensor networks with adjustable ranges // Adhoc. Sens. Networks, No. 16, 1-28, 2012.

[16] Rogers C. Packing and covering. Cambridge University Press, Cambridge, 1964.

[17] Sengupta S., Das S., Nasir M., Panigrahi B. Multi-objective node deployment in WSNs: In search of an optimal trade-off among coverage, lifetime, energy consumption, and connectivity // Engineering Applications of Artificial Intelligence, 26(1), 405-416, 2013.

[18] Ssu K.-F., Wang W.-T., Wu F.-K., Wu T.-T. K-barrier coverage with a directional sensing model // Int. J. on Smart Sensing and Intelligent Systems, 2(1), 75-93, 2009.

[19] Fejes Töth G. Covering the plane with two kinds of circles // Discrete \& Computational Geometry, No. 13, 445-457, 1995.

[20] Wang W., Srinivasan V., Wang B., Chua K.-C. Coverage for target localization in wireless sensor networks // Wireless Communications, IEEE Transactions on Wireless Communications, 7(2), 667-676, 2008.

[21] Wu J., Yang S. Energy-efficient node scheduling models in sensor networks with adjustable ranges // Int. J. of Foundations of Computer Science, 6(1), 3-17, 2005.

[22] Zalyubovskiy V., Aldyn-ool T., Erzin A., Choo H. Energy-efficient coverage-guaranteed node scheduling models in sensor networks with random deployed sensors // 2nd Int. conf. on Internet, 757-760, 2010.

[23] Zalyubovsky V., Erzin A., Astrakov S., Choo H. Energy-efficient area coverage by sensors with adjustable ranges // Sensors, 9(4), 2446-2460, 2009. 